\title{
A note on Laguerre translations
}

\author{
Günter F. Steinke
}

\begin{abstract}
Using the correspondence between $2 n$-dimensional Laguerre planes and compact antiregular generalized quadrangles with parameter $n=1,2$ we show that almost each automorphism of such a Laguerre plane that induces a translation in the derived affine plane at a point $p$ is a Laguerre translation, that is, fixes all points on the parallel class of $p$. The same is true for ovoidal Laguerre planes over ovals that also are dual ovals.
\end{abstract}

Keywords: Laguerre plane, Lie geometry, generalized quadrangle, Laguerre translation MSC 2000: 51H15, 51B15

\section{Introduction and results}

A Laguerre translation of a Laguerre plane $\mathcal{L}$ is an automorphism of $\mathcal{L}$ that fixes the points of a parallel class and induces a translation in the derived affine plane at one of its fixed points, compare [3] or [5]. Often it is clear that an automorphism of a Laguerre plane has this last property, which we refer to as the translation property at the point $p$ for short, but it usually takes some effort to verify that one has a Laguerre translation, that is, that all the points of the parallel class $|p|$ of $p$ are fixed. Hartmann [3, Lemma 2.2] obtained several rather restrictive conditions for the translation property implying Laguerre translation in a general setting.

The aim of this paper is to use the close relationship between $2 n$-dimensional Laguerre planes for $n=1,2$ and compact antiregular generalized quadrangles with parameter $n$ and give a partial answer to Problem 5.9.8 in [7] in that we show that in fact for an automorphism of such a Laguerre plane $\mathcal{L}$ not in the kernel of $\mathcal{L}$ the translation property at a point suffices in order to have a Laguerre translation. (The kernel of a Laguerre plane consists of all automorphisms that fix each parallel class and is a normal subgroup of the automorphism group.) 






